\title{
Feedback and Open-Loop Nash Equilibria in a Class of Differential Games with Random Duration *
}

\author{
Anna V. Tur and Natalya G. Magnitskaya \\ St.Petersburg State University \\ 7/9, Universitetskaya nab., Saint-Petersburg \\ 199034, Russia \\ E-mail: a.tur@spbu.ru \\ E-mail: magnitsnatalya@gmail.com
}

\begin{abstract}
One class of differential games with random duration is considered. It is assumed that duration of the game is a random variable with values from a given finite interval. The game can be interrupted only on this interval. Methods of construction feedback and open-loop Nash equilibria for such games are proposed.
\end{abstract}

Keywords: differential game, Nash equilibrium, random variable, open-loop strategies, feedback strategies

\section{Introduction}

Differential game theory is commonly used to describe realistic conflict-controlled processes with many participants. When modeling economic or environmental processes, many researchers turn to the use of differential games with finite or infinite duration. However, the recently popular direction of studying games with random duration allows to simulate a process close to the real one, in which the terminal time of the game is not known in advance, but is an implementation of some random variable (Petrosjan and Murzov, 1966; Petrosjan and Shevkoplyas, 2000; Shevkoplyas, 2014). For the first time the class of differential games with random duration was introduced in (Petrosyan and Murzov, 1966) for a particular case of a zero-sum pursuit game. Later, the general formulation of the differential games with random duration was given in (Petrosyan and Shevkoplyas, 2000).

The aim of this paper is to investigate the case when the ending of the game is possible not over the whole period of the game, but only at a certain given interval. Players know that the game will not be interrupted until a certain point. After this moment, the game may abruptly end. Consideration of the problem in this vein leads to the fact that payoffs of players can be represented as sums of integrals with different but adjoint time intervals. The paper provides ways to construct open-loop and feedback Nash equilibria in this class of games.

The paper is structured as follows. In section 2 the problem formulation is given. One method of construction feedback Nash equilibrium is considered in section 3 . This method is applied to an illustrative example in section 3.1. The construction of Nash equilibrium in open-loop strategies is investigated in section 4 . In section 4.1 open-loop Nash equilibrium strategies are studied for the example from sec. 3.1.

^ The reported study was funded by RFBR according to the research project N 18-0000727 (18-00-00725) 


\section{Problem Formulation}

Consider differential $n$-player game $\Gamma\left(x_{0}, T-t_{0}\right)$ defined on the interval $\left[t_{0}, T\right]$ with the system dynamics described by differential equations:

$$
\begin{aligned}
& \dot{x}(t)=g(t, x, u) \\
& x\left(t_{0}\right)=x_{0} \\
& x \in \mathbb{R}^{l}, u=\left(u_{1}, \ldots, u_{n}\right), u_{i}=u_{i}(t) \in U_{i} \subset \mathrm{comp} \mathbb{R}^{k}
\end{aligned}
$$

The game $\Gamma\left(x_{0}, T-t_{0}\right)$ starts from initial state $x_{0}$ at the time instant $t_{0}$. But duration of the game is not fixed. We assume that $T$ is a random variable with some predetermined distribution law. Let a cumulative distribution function has the form:

$$
F(\tau)= \begin{cases}0, & \text { for } \tau<\bar{T}-\delta \\ \varphi(\tau), & \text { for } \bar{T}-\delta \leq \tau<\bar{T}+\delta \\ 1, & \text { for } \tau \geq \bar{T}+\delta\end{cases}
$$

where $\varphi(\tau)$ is assumed to be an absolutely continuous nondecreasing function satisfying the following conditions: $\varphi(\bar{T}-\delta)=0, \varphi(\bar{T}+\delta)=1$. This means that the game could end only during the period $[\bar{T}-\delta, \bar{T}+\delta]$, where $\delta \in\left[t_{0}, \bar{T}\right]$.

The expected payoff of player $i \in N$ in $\Gamma\left(x_{0}, T-t_{0}\right)$ is defined in the following way:

$$
K_{i}\left(x_{0}, T-t_{0} ; u\right)=\int_{t_{0}}^{\bar{T}-\delta} h^{i}[s, x(s), u] d s+\int_{\bar{T}-\delta}^{\bar{T}+\delta} \int_{\bar{T}-\delta}^{\tau} h^{i}[s, x(s), u] d s d F(\tau)
$$

According to (Gromova and Tur, 2017), where the transformation procedure of the double integral functional and its reduction to a single integral is described, the expected payoff of player $i \in N$ can be represented in the form:

$$
K_{i}\left(x_{0}, T-t_{0} ; u\right)=\int_{t_{0}}^{\bar{T}-\delta} h^{i}[s, x(s), u] d s+\int_{\bar{T}-\delta}^{\bar{T}+\delta} h^{i}[s, x(s), u](1-F(s)) d s .
$$

And the expected payoff of player $i$ in subgame $\Gamma(x(t), T-t)$, starting at the moment $t$ from $x(t)$ is:

$$
\begin{aligned}
& K_{i}(x(t), T-t ; u)= \\
& =\left\{\begin{array}{l}
\int_{t}^{\bar{T}-\delta} h^{i}[s, x(s), u] d s+\int_{\bar{T}-\delta}^{\bar{T}+\delta} h^{i}[s, x(s), u](1-F(s)) d s, \text { for } t \in\left[t_{0}, \bar{T}-\delta\right), \\
\frac{1}{1-F(t)} \int_{t}^{\bar{T}+\delta} h^{i}[s, x(s), u](1-F(s)) d s, \text { for } t \in[\bar{T}-\delta, \bar{T}+\delta) .
\end{array}\right.
\end{aligned}
$$

We assume an existence of a probability density function $f(t)=F^{\prime}(t)$.

\section{Feedback Nash Equilibrium. Hamilton-Jacobi-Bellman Equations}

One of the principles of optimality in non-cooperative differential games is a feedback Nash equilibrium. Feedback Nash equilibrium strategies depend only on 
the time variable and the current value of the state, but not on memory (including the initial state $x_{0}$ ) (Başar and Olsder, 1995). We use the sufficient conditions of Hamiltom-Jacobi-Bellman equations in order to find a feedback Nash equilibrium. The Hamilton-Jacobi-Bellman equations for differential games with random duration was proposed in (Shevkoplyas, 2014). Here this method is adapted for the problem under consideration.

In the framework of this approach the Bellman function $V^{i}(t, x)$ is defined as the payoff of player $i$ in feedback Nash equilibrium $u^{N E}(t, x)$ in the subgame of $\Gamma(x(t), T-t)$ starting at the instant $t \in[\bar{T}-\delta, \bar{T}+\delta]$ in the state $x(t)$. And $W^{i}(t, x)$ is a payoff of player $i$ in feedback Nash equilibrium $u^{N E}(t, x)$ in the subgame of $\Gamma(x(t), T-t)$ starting at the instant $t \in\left[t_{0}, \bar{T}-\delta\right]$ in the state $x(t)$.

The following theorem takes place:

Theorem 1. $u^{N E}(t, x)$ is the Nash equilibrium in feedback strategies in the differential game $\Gamma\left(x_{0}, T-t_{0}\right)$, if there exist continuously differentiable functions $V^{i}(t, x):[\bar{T}-\delta, \bar{T}+\delta] \times R^{l} \rightarrow R, i \in N$ and $W^{i}(t, x):\left[t_{0}, \bar{T}-\delta\right] \times R^{l} \rightarrow R, i \in N$, satisfying the following system of partial differential equations:

$$
\begin{aligned}
& \frac{\dot{\varphi}(t)}{1-\varphi(t)} V^{i}(t, x)-V_{t}^{i}(t, x)=\max _{\phi_{i}}\left\{h^{i}\left(t, x, u_{-i}^{N E}\right)+V_{x}^{i}(t, x) g\left(t, x, u_{-i}^{N E}\right)\right\}= \\
& =h^{i}\left(t, x, u^{N E}\right)+V_{x}^{i}(t, x) g\left(t, x, u^{N E}\right), i \in N, \\
& V^{i}(\bar{T}+\delta, x(\bar{T}+\delta))=0, \quad i \in N, \\
& -W_{t}^{i}(t, x)=\max _{\phi_{i}}\left\{h^{i}\left(t, x, u_{-i}^{N E}\right)+W_{x}^{i}(t, x) g\left(t, x, u_{-i}^{N E}\right)\right\}= \\
& h^{i}\left(t, x, u^{N E}\right)+W_{x}^{i}(t, x) g\left(t, x, u^{N E}\right) i \in N, \\
& W^{i}(\bar{T}-\delta, x(\bar{T}-\delta))=V^{i}(\bar{T}-\delta, x(\bar{T}-\delta)), i \in N,
\end{aligned}
$$

where $u_{-i}^{N E}\left(\phi_{i}\right)=\left(u_{1}^{N E}, \ldots, \phi_{i}, \ldots, u_{n}^{N E}\right)$.

Proof. Define $I_{1}=\left[t_{0}, \bar{T}-\delta\right]$ and $I_{2}=[\bar{T}-\delta, \bar{T}+\delta]$.

First, consider our problem on the segment $I_{2}$. The payoff of player $i \in N$ on $I_{2}$ is given by

$K_{i}^{I_{2}}(x(t), T-t ; u)=\frac{1}{1-F(t)} \int_{t}^{\bar{T}+\delta} h^{i}[s, x(s), u](1-F(s)) d s$, for $t \in[\bar{T}-\delta, \bar{T}+\delta)$.

The Bellman function $V^{i}(t, x)$ is defined as the payoff of player $i$ in feedback Nash equilibrium $u^{N E}(t, x)$ in the subgame of $\Gamma(x(t), T-t)$ starting at the instant $t \in[\bar{T}-\delta, \bar{T}+\delta]$ in the state $x(t)$.

According to (Shevkoplyas, 2014) HJB equations for finding Nash equilibrium in the game with payoffs of the form (7) are as follows:

$$
\begin{aligned}
& \frac{\dot{\varphi}(t)}{1-\varphi(t)} V^{i}(t, x)-V_{t}^{i}(t, x)=\max _{\phi_{i}}\left\{h^{i}\left(t, x, u_{-i}^{N E}\right)+V_{x}^{i}(t, x) g\left(t, x, u_{-i}^{N E}\right)\right\}= \\
& =h^{i}\left(t, x, u^{N E}\right)+V_{x}^{i}(t, x) g\left(t, x, u^{N E}\right), i \in N, \\
& V^{i}(\bar{T}+\delta, x(\bar{T}+\delta))=0, \quad i \in N,
\end{aligned}
$$


where $u_{-i}^{N E}\left(\phi_{i}\right)=\left(u_{1}^{N E}, \ldots, \phi_{i}, \ldots, u_{n}^{N E}\right)$.

Consider now our problem starting at some moment $t \in I_{1}$. The payoff of player $i \in N$ is:

$$
K_{i}^{I_{1}}(x(t), T-t ; u)=\int_{t}^{\bar{T}-\delta} h^{i}[s, x(s), u] d s+V_{i}(\bar{T}-\delta, x(\bar{T}-\delta)),
$$

where $V_{i}(\bar{T}-\delta, x(\bar{T}-\delta))$ - the payoff of player $i$ in Nash equilibrium for the period $I_{2}$. The value $V_{i}(\bar{T}-\delta, x(\bar{T}-\delta))$ is considered as a terminal payoff of player $i$ for the period $I_{1}$. So we get the second part of (6):

$$
\begin{aligned}
& -W_{t}^{i}(t, x)=\max _{\phi_{i}}\left\{h^{i}\left(t, x, u_{-i}^{N E}\right)+W_{x}^{i}(t, x) g\left(t, x, u_{-i}^{N E}\right)\right\}= \\
& h^{i}\left(t, x, u^{N E}\right)+W_{x}^{i}(t, x) g\left(t, x, u^{N E}\right) i \in N, \\
& W^{i}(\bar{T}-\delta, x(\bar{T}-\delta))=V^{i}(\bar{T}-\delta, x(\bar{T}-\delta)), i \in N,
\end{aligned}
$$

where $u_{-i}^{N E}\left(\phi_{i}\right)=\left(u_{1}^{N E}, \ldots, \phi_{i}, \ldots, u_{n}^{N E}\right)$.

\subsection{Differential Game of Investment}

Consider an illustrative example. Assume that there are $n$ individuals who invest in a public stock of knowledge (Dockner et al., 2000). Let $x(t)$ be the stock of knowledge at time $t$ and $u_{i}(t, x)$ - the investment of agent $i$ in public knowledge at time $t$. The stock of knowledge evolves according to the accumulation equation:

$$
\dot{x}(t)=u_{1}(t)+u_{2}(t)+\ldots+u_{n}(t), \quad x(0)=x_{0} .
$$

If each agent derives linear utility from the consumption of the stock of knowledge, the expected payoff of player $i \in N$ is given by

$$
K_{i}\left(x_{0}, T ; u\right)=E \int_{0}^{T}\left(q_{i} x(t)-r_{i} u_{i}^{2}(t)\right) d t .
$$

Assume that the random variable $\mathrm{T}$ distributed uniformly on $[\bar{T}-\delta, \bar{T}+\delta]$. The cumulative distribution function has the form:

$$
F(\tau)= \begin{cases}0, & \text { for } \tau<\bar{T}-\delta \\ \frac{t-\bar{T}+\delta}{2 \delta}, & \text { for } \bar{T}-\delta \leq \tau<\bar{T}+\delta, \\ 1, & \text { for } \tau \geq \bar{T}+\delta\end{cases}
$$

\subsection{Feedback Nash Equilibrium}

To find the feedback Nash equilibrium in the subgame, starting at the time instant $\bar{T}-\delta$ from the state $x(\bar{T}-\delta)$, consider the first part of HJB equations (6):

$$
\begin{aligned}
& \frac{1}{\bar{T}+\delta-t} V^{i}(t, x)-V_{t}^{i}(t, x)=\max _{u_{i}}\left(q_{i} x-r_{i} u_{i}^{2}+V_{x}^{i}(t, x)\left(u_{i}+\sum_{j \neq i} u_{j}^{N E}\right)\right), \\
& V_{i}(\bar{T}+\delta, x(\bar{T}+\delta))=0, \quad i \in N .
\end{aligned}
$$

Bellman function is defined in the form: $V^{i}(t, x)=a_{i}(t) x+b_{i}(t)$. The maximization problem in (14) yields a strategy for player $i$ :

$$
u_{i}^{N E}(t, x)=\frac{V_{x}^{i}(t, x)}{2 r_{i}}=\frac{a_{i}(t)}{2 r_{i}} .
$$


Substituting it into (14) we have the following system of differential equations for $a_{i}(t)$ :

$$
\begin{gathered}
\dot{a}_{i}(t)=\frac{1}{\bar{T}+\delta-t} a_{i}(t)-q_{i}, \quad i \in N, \\
a_{i}(\bar{T}+\delta)=0 .
\end{gathered}
$$

Then

$$
a_{i}(t)=\frac{q_{i}}{2}(\bar{T}+\delta-t)
$$

For $b_{i}(t)$ we have:

$$
\begin{gathered}
\dot{b}_{i}(t)=\frac{1}{\bar{T}+\delta-t} b_{i}(t)-\frac{a_{i}^{2}}{4 r_{i}}-\sum_{i \neq j} \frac{a_{i} a_{j}}{2 r_{j}}, \quad i \in N, \\
b_{i}(\bar{T}+\delta)=0 .
\end{gathered}
$$

So we get

$$
b_{i}(t)=\frac{m_{i}}{4}(\bar{T}+\delta-t)^{3}, \quad i \in N,
$$

where $m_{i}=\frac{q_{i}^{2}}{16 r_{i}}+\sum_{i \neq j} \frac{q_{i} q_{j}}{8 r_{j}}$.

Then the feedback Nash equilibrium strategies on $I_{2}$ are:

$$
u_{i}^{N E}(t, x)=\frac{q_{i}}{4 r_{i}}(\bar{T}+\delta-t), \quad i=1 \in N, t \in[\bar{T}-\delta, \bar{T}+\delta] .
$$

The payoff of player $i \in N$ in Nash equilibrium for the period $I_{2}$ looks as follows:

$$
V_{i}(\bar{T}-\delta, x(\bar{T}-\delta))=q_{i} \delta x(\bar{T}-\delta)+2 m_{i} \delta^{3}, \quad i \in N .
$$

Then, the bound condition for the problem on $I_{1}$ is:

$$
W_{i}(\bar{T}-\delta, x(\bar{T}-\delta))=q_{i} \delta x(\bar{T}-\delta)+2 m_{i} \delta^{3}, \quad i \in N .
$$

To find the feedback Nash equilibrium in the subgame, starting at the time instant $t_{0}$ from $x_{0}$ and ending at $\bar{T}-\delta$ in $x(\bar{T}-\delta)$, consider the system of HJB equations:

$$
\begin{gathered}
-W_{t}^{i}(t, x)=\max _{u_{i}}\left(q_{i} x-r_{i} u_{i}^{2}+W_{x}^{i}(t, x)\left(u_{i}+\sum_{j \neq i} u_{j}^{N E}\right)\right), \quad i \in N, \\
W^{i}(\bar{T}-\delta, x(\bar{T}-\delta))=q_{i} \delta x(\bar{T}-\delta)+2 m_{i} \delta^{3}, i \in N .
\end{gathered}
$$

Bellman function is defined in the form: $W^{i}(t, x)=c_{i}(t) x+d_{i}(t)$. The maximization problem in (19) yields a strategy for player $i: u_{i}^{N E}(t, x)=\frac{W_{x}^{i}(t, x)}{2 r_{i}}=\frac{c_{i}(t)}{2 r_{i}}$. Substituting it into (19) we have the following system of differential equations for $c_{i}(t)$ :

$$
\begin{gathered}
\dot{c}_{i}(t)=-q_{i}, \quad i \in N \\
c_{i}(\bar{T}-\delta)=q_{i} \delta .
\end{gathered}
$$

Then

$$
c_{i}(t)=q_{i}(\bar{T}-t)
$$


For $d_{i}(t)$ we have:

$$
\begin{gathered}
\dot{d}_{i}(t)=-\frac{c_{i}^{2}}{4 r_{i}}-\sum_{i \neq j} \frac{c_{i} c_{j}}{2 r_{j}}, \quad i \in N, \\
d_{i}(\bar{T}-\delta)=2 m_{i} \delta^{3} .
\end{gathered}
$$

We get

$$
d_{i}(t)=\frac{4 m_{i}(\bar{T}-t)^{3}}{3}+\frac{2 m_{i} \delta^{3}}{3}, \quad i \in N,
$$

where $m_{i}=\frac{q_{i}^{2}}{16 r_{i}}+\sum_{i \neq j} \frac{q_{i} q_{j}}{8 r_{j}}$.

Then the feedback Nash equilibrium strategies in the game $\Gamma\left(x_{0}, T-t_{0}\right)$ are:

$$
u_{i}^{N E}(t)=\left\{\begin{array}{l}
\frac{q_{i}}{2 r_{i}}(\bar{T}-t), \quad i=1 \in N, t \in\left[t_{0}, \bar{T}-\delta\right] \\
\frac{q_{i}}{4 r_{i}}(\bar{T}+\delta-t), \quad i=1 \in N, t \in[\bar{T}-\delta, \bar{T}+\delta]
\end{array}\right.
$$

\section{Open-Loop Nash Equilibrium}

The second part of the paper is devoted to construction of open-loop Nash equilibrium for the game under consideration, which depends only on the time parameter $t$ and the initial state of the system (Başar and Olsder, 1995).

The method we introduce here is based on Pontryagin's maximum principle (Pontryagin et al., 1963).

We will find the solution on two intervals $I_{1}=\left[t_{0} ; \bar{T}-\delta\right]$ and $I_{2}=[\bar{T}-\delta ; \bar{T}+\delta]$. The boundary conditions at time $T-\delta$ are considered as parameters. We will find their values at the end of the solution from the maximization condition.

Let's start with studying the game at the period $I_{1}$.

Each player $i \in N$ tries to maximize $\int_{t}^{\bar{T}-\delta} h^{i}[s, x(s), u] d s$ for dynamic (1). The problem will be solved with two fixed ends: $x\left(t_{0}\right)=x_{0}$ and $x(\bar{T}-\delta)=x_{1}$. Introduce $x_{1}$ as a parameter of the solution (we will see that $x_{1}$ is indeed a function of $n$ parameters). The use of such a method for cooperative differential games was proposed in (Gromov and Gromova, 2017), (Gromova and Magnitskaya, 2019). Here we adapt it for non-cooperative games.

On the interval $I_{1}$ the Hamiltonian for player $i$ is:

$$
H_{i}\left(x, u_{-i}^{N E}, \psi\right)=\psi_{i} g\left(t, x, u_{-i}^{N E}\right)+h^{i}\left(t, x, u_{-i}^{N E}\right), i \in N .
$$

The equilibrium strategies $u_{i}^{N E}$ are found from the first order extremality condition:

$$
\frac{\partial H_{i}\left(x, u_{-i}^{N E}, \psi\right)}{\partial u_{i}}=0 .
$$

The adjoint equations are:

$$
\frac{\partial \psi_{i}}{\partial t}=-\frac{\partial H_{i}\left(x, u^{N E}, \psi\right)}{\partial x} .
$$


We introduce the boundary conditions $\psi_{i}(\bar{T}-\delta)=z_{i}$ as parameters of the solution. Let $u_{I_{1}}^{N E}\left(s, z_{1}, \ldots, z_{n}\right)$ - equilibrium strategies on $I_{1}$. And the equilibrium trajectory $x_{I_{1}}^{N E}\left(t, z_{1}, \ldots, z_{n}\right)$ we can found from (1). And $x_{1}=x_{I_{1}}^{N E}\left(\bar{T}-\delta, z_{1}, \ldots, z_{n}\right)$.

Now turn to studying the solution on the second interval $I_{2}$.

Player $i$ maximizes $\int_{\bar{T}-\delta}^{\bar{T}+\delta} h^{i}[s, x(s), u](1-F(s)) d s$ for dynamic (1) with initial condition $x(\bar{T}-\delta)=x_{1}=x_{I_{1}}^{N E}\left(\bar{T}-\delta, z_{1}, \ldots, z_{n}\right)$, and with a loose right end.

The Hamiltonian for player $i$ is:

$$
H_{i}\left(x, u_{-i}^{N E}, \psi\right)=\psi_{i} g\left(t, x, u_{-i}^{N E}\right)+(1-F(t)) h^{i}\left(t, x, u_{-i}^{N E}\right) .
$$

To find equilibrium strategies $u_{i}^{N E}$ we use the necessary condition for the maximum:

$$
\frac{\partial H_{i}\left(x, u_{-i}^{N E}, \psi\right)}{\partial u_{i}}=0
$$

The adjoint equations are:

$$
\frac{\partial \psi_{i}}{\partial t}=-\frac{\partial H_{i}\left(x, u^{N E}, \psi\right)}{\partial x}
$$

with transversality conditions

$$
\psi_{i}(\bar{T}+\delta)=0
$$

Let $u_{I_{2}}^{N E}\left(t, z_{1}, \ldots, z_{n}\right)$ - equilibrium strategies on $I_{2}$ and $x_{I_{2}}^{N E}\left(t, z_{1}, \ldots, z_{n}\right)$ equilibrium trajectory on $I_{2}$.

On the last step of our solution we find the value of parameters $z_{1}^{*}, \ldots, z_{n}^{*}$ in the following way:

$$
\begin{aligned}
z_{i}^{*}=\arg \max _{z_{i}}\left(\int_{t_{0}}^{\bar{T}-\delta} h^{i}\left[s, x_{I_{1}}^{N E}\left(s, z *_{-i}\right), u_{I_{1}}^{N E}\left(s, z_{-i}^{*}\right)\right] d s+\right. \\
\left.\quad+\int_{\bar{T}-\delta}^{\bar{T}+\delta} h^{i}\left[s, x_{I_{2}}^{N E}\left(s, z_{-i}^{*}\right), u_{I_{2}}^{N E}\left(s, z_{-i}^{*}\right)\right](1-F(s)) d s\right),
\end{aligned}
$$

where $z_{-i}^{*}=\left(z_{1}^{*}, \ldots, z_{i-1}^{*}, z_{i}, z_{i-1}^{*}, \ldots, z_{n}^{*}\right)$.

Finally we get equilibrium strategies:

$$
u_{i}^{N E}(t)= \begin{cases}u_{i}^{N E}\left(t, z_{1}^{*}, \ldots, z_{n}^{*}\right)_{I_{1}}, & t \in\left[t_{0}, \bar{T}-\delta\right], \\ u_{i}^{N E}\left(t, z_{1}^{*}, \ldots, z_{n}^{*}\right)_{I_{2}}, & t \in[\bar{T}-\delta, \bar{T}+\delta] .\end{cases}
$$

The method of constructing strategies (28) ensures that they are equilibrium strategies. 


\subsection{Differential Game of Investment. Open-Loop Nash Equilibrium}

Consider again the example suggested in section 3 . Find the solution in the class of open-loop strategies.

Intervals $I_{1}$

Let's start with studying the game at the period $I_{1}$.

Payoff of player $i$ on $I_{1}$ is $\int_{0}^{\bar{T}-\delta}\left(q_{i} x(t)-r_{i} u_{i}^{2}(t)\right) d t$ for dynamic (1).

The Hamiltonian for player $i$ is:

$$
\left.H_{i}(x, u, \psi)=\psi_{i}\left(u_{i}+\sum_{j \neq i} u_{j}^{N E}\right)\right)+q_{i} x(t)-r_{i} u_{i}^{2}(t) .
$$

To find equilibrium strategies $u_{i}^{N E}$ we use the necessary condition for the maximum:

$$
\begin{gathered}
\frac{\partial H_{i}}{\partial u_{i}}=\psi_{i}-2 r_{i} u_{i}(t)=0, \\
u_{i}^{N E}(t)=\frac{\psi_{i}}{2 r_{i}} .
\end{gathered}
$$

The Hessian matrix is negative definite hence we conclude that Hamiltonian $H_{i}$ is concave w.r.t. $u_{i}, t \in[0, \bar{T}-\delta]$,

$$
\frac{\partial^{2} H_{i}}{\partial u_{i}^{2}}=-2 r_{i}<0
$$

The adjoint equations are as follows:

$$
\frac{\partial \psi_{i}}{\partial t}=-\frac{\partial H_{i}(x, u, \psi)}{\partial x}=-q_{i} .
$$

We introduce the boundary conditions $\psi_{i}(\bar{T}-\delta)=z_{i}, i=1, \ldots, n$ as parameters of the solution.

Hence,

$$
\begin{gathered}
\psi_{i}(t)=z_{i}-q_{i} t, \\
u_{i}^{N E}(t, z)_{I_{1}}=\frac{z_{i}-q_{i} t}{2 r_{i}} .
\end{gathered}
$$

The dynamic is:

$$
\dot{x}(t)=\sum_{i=1}^{n} u_{i}(t)=\sum_{i=1}^{n} \frac{z_{i}-q_{i} t}{2 r_{i}}=z-t \tilde{q},
$$

where $z=\sum_{i=1}^{n} \frac{z_{i}}{2 r_{i}}, \tilde{q}=\sum_{i=1}^{n} \frac{q_{i}}{2 r_{i}}$.

We use the boundary condition:

$$
x(0)=x_{0} .
$$

Then the optimal trajectory for the interval $I_{1}$ :

$$
x_{I_{1}}^{N E}(t, z)=z t-\frac{\tilde{q} t^{2}}{2}+x_{0} .
$$


Then $x_{I_{1}}^{N E}(\bar{T}-\delta, z)=z(\bar{T}-\delta)-\frac{\tilde{q}(\bar{T}-\delta)^{2}}{2}+x_{0}$.

Intervals $I_{2}$

Now turn to studying the solution on the second interval $I_{2}$.

Player $i$ maximizes $\int_{\bar{T}-\delta}^{\bar{T}+\delta}\left(1-\frac{t-\bar{T}+\delta}{2 \delta}\right)\left(q_{i} x(t)-r_{i} u_{i}^{2}(t)\right) d t$ for dynamic (1) and initial condition $x(\bar{T}-\delta)=x_{I_{1}}^{N E}\left(\bar{T}-\delta, z_{1}, \ldots, z_{n}\right)$.

The Hamiltonian for player $i$ is:

$$
\left.H_{i}(x, u, \phi)=\phi_{i}\left(u_{i}+\sum_{j \neq i} u_{j}^{N E}\right)\right)+\left(1-\frac{t-\bar{T}+\delta}{2 \delta}\right)\left(q_{i} x(t)-r_{i} u_{i}^{2}(t)\right) .
$$

To find equilibrium strategies $u_{i}^{N E}$ we use the necessary condition for the maximum:

$$
\begin{gathered}
\frac{\partial H_{i}}{\partial u_{i}}=\phi_{i}-2\left(1-\frac{t-\bar{T}+\delta}{2 \delta}\right) r_{i} u_{i}=0, \\
u_{i}^{N E}(t)_{I_{2}}=\frac{\phi_{i} \delta}{(\delta-t+\bar{T}) r_{i}} .
\end{gathered}
$$

The Hessian matrix is negative definite hence we conclude that Hamiltonian $H_{i}$ is concave w.r.t. $u_{i}, t \in[\bar{T}-\delta ; \bar{T}+\delta]$,

$$
\frac{\partial^{2} H_{i}}{\partial u_{i}^{2}}=-2\left(1-\frac{t-\bar{T}+\delta}{2 \delta}\right) r_{i}<0 .
$$

The adjoint equations are:

$$
\frac{\partial \phi_{i}}{\partial t}=-\frac{\partial H_{i}(x, u, \phi)}{\partial x}=-\left(1-\frac{t-\bar{T}+\delta}{2 \delta}\right) q_{i}
$$

with transversality conditions:

$$
\phi_{i}(\bar{T}+\delta)=0, \quad i \in N .
$$

We get the solution of (35)-(36):

$$
\phi_{i}=-\frac{q_{i}(\delta+\bar{T})(t-\bar{T}+\delta)}{2 \delta}+\frac{q_{i}\left(t^{2}-(\bar{T}-\delta)^{2}\right)}{4 \delta}+q_{i} \delta .
$$

To get the optimal trajectory substitute (37) into (11):

$$
\dot{x}(t)=\frac{\delta}{t-\bar{T}+\delta} \sum_{i=1}^{n} \frac{\phi_{i}}{r_{i}} .
$$

The boundary condition is the following: $x(\bar{T}-\delta)=x_{I_{1}}^{N E}\left(\bar{T}-\delta, z_{1}, \ldots, z_{n}\right)=$ $z(\bar{T}-\delta)-\frac{\tilde{q}(\bar{T}-\delta)^{2}}{2}+x_{0}$.

Then the optimal trajectory is:

$$
x_{I_{2}}^{N E}(t)=z(\bar{T}-\delta)-\frac{\tilde{q}(\bar{T}-\delta)^{2}}{2}+x_{0}+C(t),
$$

where $C(t)$ is an expression independent of $z_{1}, \ldots, z_{n}$. 
Optimal strategies on $I_{2}$ have the form:

$$
\begin{gathered}
u_{i}^{N E}(t)_{I_{2}}=\left(-\frac{q_{i}(\delta+\bar{T})(t-\bar{T}+\delta)}{2 \delta}+\frac{q_{i}\left(t^{2}-(\bar{T}-\delta)^{2}\right)}{4 \delta}+q_{i} \delta\right) \frac{\delta}{(\delta-t+\bar{T}) r_{i}}= \\
=\frac{q_{i}(\bar{T}+\delta-t)}{4 r_{i}} .
\end{gathered}
$$

Intervals $I_{1}, I_{2}$

We find $z_{1}, \ldots, z_{n}$ from the maximization condition of the total payoff in the interval $[0, \bar{T}+\delta]$, i.e.

$$
z_{i}^{*}=\arg \max _{z_{i}} K_{i}\left(0, x_{0}, u^{N E}\left(t, z_{-i}^{*}\right)\right),
$$

according to the (33), (31), (38), (39). Substituting (33), (31), (38), (39) to the (27), we get:

$$
\begin{gathered}
\int_{0}^{\bar{T}-\delta}\left(q_{i} x^{N E}(t)_{I_{1}}-r_{i} u_{i}^{N E}(t)_{I_{1}}^{2}\right) d t+\int_{\bar{T}-\delta}^{\bar{T}+\delta}\left(1-\frac{t-\bar{T}+\delta}{2 \delta}\right)\left(q_{i} x^{N E}(t)_{I_{2}}-r_{i} u_{i}^{N E}(t)_{I_{2}}^{2}\right) d t= \\
\quad=\frac{q_{i} z(\bar{T}-\delta)^{2}}{2}-\frac{z_{i}^{2}(\bar{T}-\delta)}{4 r_{i}}+\frac{q_{i} z_{i}(\bar{T}-\delta)^{2}}{4 r_{i}}+q_{i} z(\bar{T}-\delta) \delta+B(\bar{T}, \delta),
\end{gathered}
$$

where $B(\bar{T}, \delta)$ is an expression independent of $z_{1}, \ldots, z_{n}$.

To find $z_{i}^{*}$ we use the necessary condition for the maximum:

$$
\frac{\partial K_{i}}{\partial z_{i}}=0, i=1, \ldots, n
$$

Solving

$$
\frac{q_{i}(\bar{T}-\delta)^{2}}{4 r_{i}}-\frac{2 z_{i}(\bar{T}-\delta)}{4 r_{i}}+\frac{q_{i}(\bar{T}-\delta)^{2}}{4 r_{i}}+\frac{q_{i}(\bar{T}-\delta) \delta}{2 r_{i}}=0, i=1, \ldots, n,
$$

we have:

$$
z_{i}^{*}=q_{i} \bar{T}
$$

Finally, we get

$$
\begin{gathered}
u_{i}^{N E}(t)_{I_{1}}=\frac{q_{i}(\bar{T}-t)}{2 r_{i}}, \quad t \in[0, \bar{T}-\delta], \\
u_{i}^{N E}(t)_{I_{2}}=\frac{q_{i}(\bar{T}+\delta-t)}{4 r_{i}}, \quad t \in[\bar{T}-\delta, \bar{T}+\delta] .
\end{gathered}
$$

It can be noted, that in the game under consideration open-loop and feedback equilibrium strategies coincide. This is also characteristic of classical differential games with a linear structure.

Note also that $\lim _{\delta \rightarrow 0} u_{i}^{N E}(t)=\frac{q_{i}(\bar{T}-t)}{2 r_{i}}$, and these are Nash equilibrium strategies in the differential game with prescribed duration $\bar{T}-t_{0}$. 


\subsection{Numeric Example}

Consider the previous example with numeric parameters.

Let $n=3, \bar{T}=10, \delta=3, q_{1}=4, q_{2}=3, q_{3}=6, r_{1}=2, r_{2}=1, r_{3}=5, x_{0}=20$.

Consequently:

$$
\begin{gathered}
x_{I_{1}}^{N E}(t)=\frac{-31 t^{2}}{20}+31 t+20, \\
u_{1}^{N E}(t)_{I_{1}}=10-t, \\
u_{2}^{N E}(t)_{I_{1}}=15-\frac{3 t}{2}, \\
u_{3}^{N E}(t)_{I_{1}}=6-\frac{3 t}{5}, \quad t \in[0,7), \\
x_{I_{2}}^{N E}(t)=\frac{-31 t^{2}}{40}+\frac{403 t}{20}+57.975, \\
u_{1}^{N E}(t)_{I_{2}}=\frac{13}{2}-\frac{t}{2}, \\
u_{2}^{N E}(t)_{I_{2}}=\frac{39}{4}-\frac{3 t}{4}, \\
u_{3}^{N E}(t)_{I_{2}}=\frac{39}{10}-\frac{3 t}{10}, \quad t \in[7,13] .
\end{gathered}
$$

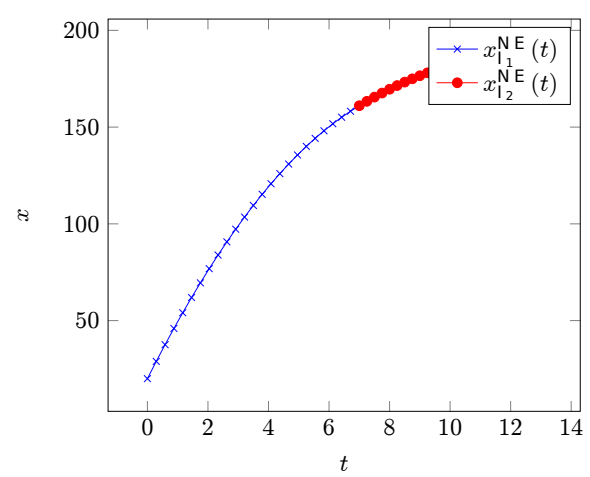

Fig. 1. Nash equilibrium trajectory

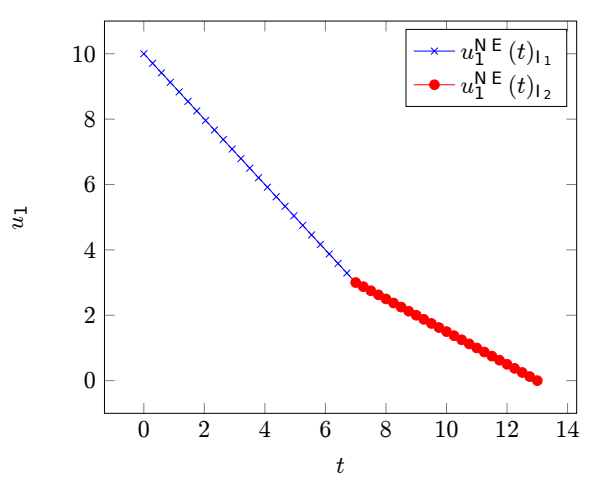

Fig. 2. Equilibrium strategy for player 1

\section{Conclusion}

The special class of differential games with random duration is investigated. The method of construction feedback Nash equilibrium based on Hamilton-JacobiBellman equations is proposed. The method of construction open-loop Nash equilibrium based on Pontryagin's maximum principle is studied. An illustrative example demonstrating both of two methods is considered. The numerical example is given. The results are drawn. 


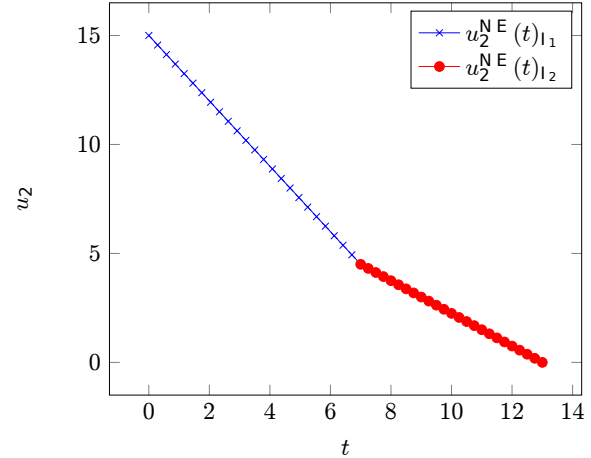

Fig. 3. Equilibrium strategy for player 2

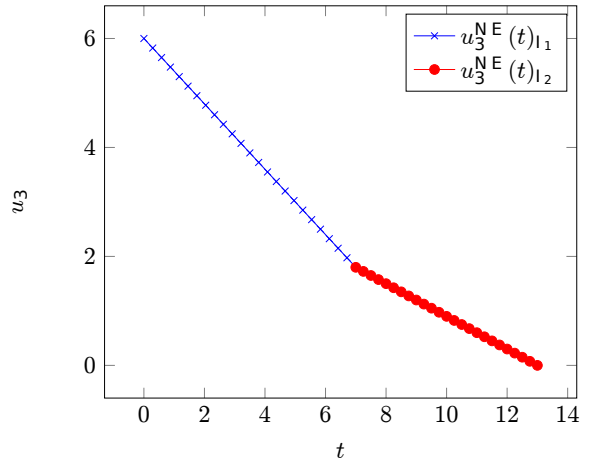

Fig. 4. Equilibrium strategy for player 3

\section{References}

Başar, T., Olsder, G. (1995). Dynamic Non-Cooperative Game Theory. London: Academic Press.

Dockner, E. J., S. Jorgensen, N. van Long, and G. Sorger (2000). Differential Games in Economics and Management Science. Cambridge Univ. Press.

Gromov, D., Gromova, E. (2017). On a Class of Hybrid Differential Games. Dyn. Games Appl. 7, 266-288. https://doi.org/10.1007/s13235-016-0185-3

Gromova, E. V., Magnitskaya, N.G. (2019). Solution of the differential game with hybrid structure. Contributions to Game Theory and Management, 12, 159-176

Gromova, E., Tur, A. (2017). On the form of integral payoff in differential games with random duration. 2017 XXVI International Conference on Information, Communication and Automation Technologies (ICAT), Sarajevo, 2017, pp. 1-6, doi: 10.1109/ICAT.2017.8171597.

Petrosyan, L. A., Murzov, N. V. (1966). Game-theoretic Problems in Mechanics. Lithuanian Mathematical Collection, 3, 423-433.

Petrosyan, L. A., Shevkoplyas, E. V. (2000). Cooperative differential games with random duration. Vestnik Sankt-Peterburgskogo Universiteta. Ser 1. Matematika Mekhanika Astronomiya Issue 4, pp. 18-23.

Pontryagin, L. S., V. G. Boltyanskii, R. V. Gamkrelidze, and E. F. Mishchenko (1963). The Mathematical Theory of Optimal Processes. Wiley-Interscience.

Shevkoplyas, E. V. (2014). The Hamilton-Jacobi-Bellman equation for a class of differential games with random duration. Autom. Remote Control, 75, 959-970. https://doi.org/10.1134/S0005117914050142 